\title{
Atendimento em voz no Ambulatório de Fonoaudiologia do Hospital das Clínicas da Universidade Federal de Minas Gerais
}

\author{
Voice disorder clinic in the speech therapy outpatient unit \\ at "Hospital das Clínicas da Universidade Federal \\ de Minas Gerais"
}

Letícia Neiva de Menezes $^{1}$
Mara Behlau ${ }^{2}$
Ana Cristina Côrtes Gama ${ }^{1}$
Letícia Caldas Teixeira $^{1}$

${ }^{1}$ Departamento de

Fonoaudiologia, Faculdade de Medicina, Universidade Federal de Minas Gerais. Av. Montese 965/302, Santa Branca. 31565-150 Belo Horizonte MG.

leticianeiva@click21.com.br

${ }^{2}$ Centro de Estudos da Voz.
Abstract This study set out to describe the profile of the patients with voice complaints and/or alterations who were attended between July 2003 and December 2006 at the speech therapy outpatient unit of "Hospital das Clinicas da Universidade Federal de Minas Gerais." This was analyzed according to age, sex, profession, schooling, origin, treatment performed and factors that interfere in medical care evolution. This was a retrospective study based on the analysis of the recorded data of the individuals according to a specifically prepared Protocol. The results showed that the individuals included in this study were predominantly young women, referred by a specialist from Belo Horizonte, who do not work and also have incomplete elementary school education. Attendance of those patients was on an individual basis, and almost half of them were discharged by the speech therapist. The elderly patients, who have functional or psychogenic dysphonia and a neutral or wheezy type of voice, showed a close correlation with the discharge rate by speech therapist. The younger individuals with organo-functional dysphonia bore a statistical correlation with abandoning treatment. The individuals who eventually abandoned treatment did not bear any correlation with the crossed variables.

Key words Voice, Records, Evaluation of health services, Public health.
Resumo O presente estudo caracterizou o perfil dos pacientes com queixas e/ou alterações vocais atendidos de julho de 2003 a dezembro de 2006 no ambulatório de fonoaudiologia do Hospital das Clínicas da Universidade Federal de Minas Gerais de acordo com as variáveis idade, sexo, profissão, escolaridade, procedência, tratamento realizado e fatores interferentes na evolução do atendimento. Trata-se de um estudo retrospectivo baseado na análise dos dados dos prontuários, coletados de acordo com protocolo especialmente elaborado. Os resultados apontaram que os individuos estudados eram predominantemente mulheres jovens, encaminhadas para o atendimento fonoaudiológico por um médico especialista, procedentes de Belo Horizonte, sem uma ocupação profissional e com ensino fundamental incompleto. $O$ atendimento fonoaudiológico aconteceu de forma individual e quase metade dos pacientes recebeu alta. Os indivíduos mais idosos, com disfonia funcional ou psicogênica e com voz do tipo neutra ou soprosa apresentaram correlação com alta fonoaudiológica. Os indivíduos mais jovens e com disfonia organofuncional tiveram correlação estatística com o desligamento do serviço. Os sujeitos que evoluíram para abandono não apresentaram correlação com as variáveis cruzadas.

Palavras-chave Voz, Registros, Avaliação de serviços de saúde, Saúde coletiva 


\section{Introdução}

As ações de saúde no Brasil originaram-se da necessidade de se resolver os problemas de saúde das classes menos privilegiadas. O Sistema Único de Saúde (SUS) foi criado em 1988, com o objetivo de ser um sistema único e integrado por uma rede regionalizada de ações e serviços, que visa a redução de doenças e o acesso universal e igualitário pela população ${ }^{1}$.

As três esferas do governo - federal, estadual e municipal - devem atuar de forma integrada e harmônica, compondo sistemas locais e/ou regionais nos quais as ações e os serviços oferecidos sejam organizados a partir de uma base de serviços menos complexa, estabelecendo referências e contra-referências. É importante que o SUS inicie sua organização pelos sistemas locais de saúde para se aproximar da população a qual se destina de modo a atender as melhor reais necessidades diagnosticadas ${ }^{2}$.

As entidades que regulam e as que oferecem serviços de saúde têm demonstrado certa preocupação com a qualidade da atenção. O foco já não é apenas atender as necessidades básicas, mas acrescentar melhorias e resolver buscando contemplar integralmente os verdadeiros princípios do SUS. Para avaliar se estes princípios estão sendo seguidos, alguns enfoques são utilizados na avaliação, dentre os quais destacam-se a estrutura dos locais de atendimento, a atenção dispensada pelos profissionais e suas consequências $^{3}$. Assim, os serviços de saúde devem ser organizados segundo as prioridades da população que os utiliza, as condições de atendimento da unidade e as peculiaridades e a dinamicidade do local.

Nas décadas de 70 e 80, os fonoaudiólogos foram inseridos nos serviços públicos pelas secretarias de educação e saúde. O primeiro movimento da área restringiu-se a apenas ocupar um lugar físico na saúde pública. O local de atendimento fonoaudiológico era estruturado como um consultório particular com o objetivo de reabilitar os pacientes. Entretanto, este tipo de atuação acarretava longas filas de espera nos serviços e mostrou-se insuficiente para suprir a demanda pelos serviços fonoaudiológicos, além de não favorecer a intervenção rápida e precoce ${ }^{4}$.

Em geral, os serviços de fonoaudiologia em instituições públicas sustentam-se em triagem, avaliação e tratamento, mas nem sempre tais abordagens são suficientes para atender toda a demanda do serviço público. Alguns trabalhos propuseram outras formas de atuação com o objetivo de agilizar o fluxo dos pacientes e permitir maior acesso aos serviços necessários. Algumas experiências relataram resultados positivos com a criação de grupos de orientação a famílias de crianças com queixas fonoaudiológicas. O objetivo dos grupos foi manter-se como porta de entrada do sistema de saúde e reduzir a lista de espera ${ }^{5}$.

Pesquisas no campo da fonoaudiologia comunitária ou coletiva tornam-se relevantes para promover medidas fonoaudiológicas de abrangência significativa na promoção da saúde da população e assim também definir melhor o papel e o lugar do fonoaudiólogo. Para tanto, os estudos devem coletar e analisar informações dos serviços existentes e promover uma análise crítica dos pontos positivos e negativos do serviço. Em consonância a estas idéias, acredita-se que a formação de futuros profissionais deve incluir, além da educação multidisciplinar, métodos e técnicas inovadoras, eficientes e de baixo custo voltadas para a realidade da saúde pública.

Neste contexto, evidencia-se a importância de se instrumentalizar programas para otimizar os serviços públicos de saúde, especialmente na fonoaudiologia, em que a demanda é cada vez maior e as experiências anteriores com práticas tradicionais têm demonstrado abrangência limitada e custo elevado. O planejamento e a implementação de programas de educação em saúde devem fundamentar-se nas necessidades da população alvo $^{2}$. Assim, conhecer características da população e o perfil epidemiológico em determinada área é o primeiro passo para se elaborar políticas voltadas para o atendimento, além de ser uma forma útil de se encontrar as deficiências do sistema e as necessidades de melhoria.

O Hospital das Clínicas (HC) de Belo Horizonte é um hospital universitário, público e geral que realiza atividades de ensino, pesquisa e assistência, sendo referência no sistema municipal e no estadual de saúde, e também no atendimento aos pacientes portadores de patologias de média e de alta complexidade. Integrado ao SUS, o HC atende a uma clientela universalizada e, sendo uma unidade especial da UFMG, é campo de ensino para cursos de medicina, enfermagem, farmácia, fisioterapia, terapia ocupacional, psicologia, nutrição e fonoaudiologia. A participação do estudante no universo hospitalar proporciona um aprendizado de qualidade, principalmente na área de saúde pública e assistência, e coloca o $\mathrm{HC}$ em destaque pelas atividades de ensino e pesquisa.

$\mathrm{O}$ ambulatório de fonoaudiologia faz parte do complexo do Hospital das Clínicas. O serviço 
iniciou seu funcionamento em julho de 2003 tendo como funções principais proporcionar a prática clínica aos acadêmicos do curso de fonoaudiologia da UFMG e ampliar a rede de assistência fonoaudiológica pelo sistema público de saúde. Neste local os alunos realizam atendimento clínico em todas as áreas da fonoaudiologia: fala, linguagem, motricidade orofacial, audição e voz.

Para compreender como o atendimento em voz vem acontecendo neste serviço, o presente estudo buscou informações sobre o atendimento dos indivíduos com queixas e/ou alterações vocais atendidos de julho de 2003 a dezembro 2006 no ambulatório de fonoaudiologia do Hospital das Clínicas da Universidade Federal de Minas Gerais. Foram consideradas as variáveis: idade, sexo, profissão, escolaridade e procedência. A avaliação vocal e o tratamento realizado foraminvestigados, e identificados os fatores que interferiram na evolução do atendimento.

O objetivo do presente trabalho foi compreender e caracterizar como essa população é atendida de modo a incorporar conhecimentos importantes para atender integralmente as diretrizes do SUS e exercer de maneira plena o papel social da fonoaudiologia no serviço público de saúde.

\section{Métodos}

O presente estudo teve como base uma análise retrospectiva dos dados dos prontuários de indivíduos com queixas e/ou alterações vocais atendidos no ambulatório de fonoaudiologia do Hospital das Clínicas da Universidade Federal de Minas Gerais, cuja primeira avaliação ocorreu no período de julho de 2003 a dezembro de 2006.

$\mathrm{O}$ ambulatório de fonoaudiologia do Hospital das Clínicas da Universidade Federal de Minas Gerais está situado em Belo Horizonte e atende por meio do SUS. O serviço teve início em julho de 2003, com o objetivo de oferecer estágio curricular aos acadêmicos em fonoaudiologia da UFMG e ampliar a rede de atendimento fonoaudiológico pelo sistema público de saúde. Estes são semanais, com 40 minutos de duração e realizados por alunos do $5^{\circ}$ ao $8^{\circ}$ períodos, supervisionados por professores do curso de fonoaudiologia da UFMG.

Inicialmente, foram incluídos na coleta dos dados todos os prontuários existentes no ambulatório de fonoaudiologia no período estipulado. Da coleta inicial, foram excluídos aqueles de indivíduos que não apresentavam queixas e/ou alterações vocais. Assim, os adequados aos critérios de inclusão do estudo foram analisados conforme um protocolo especificamente elaborado. O protocolo foi desenvolvido considerando os três aspectos principais a serem avaliados: 1) a identificação dos sujeitos; 2) características do atendimento realizado no ambulatório de fonoaudiologia; e 3) caracterização vocal dos pacientes atendidos que apresentavam queixas e/ou alterações vocais.

A primeira parte do protocolo contém informações para a identificação geral do paciente, como idade, sexo, profissão, escolaridade e procedência. A segunda parte foi utilizada para analisar o atendimento fonoaudiológico, buscando dados acerca da origem do encaminhamento ao ambulatório, o tipo de atendimento realizado, a evolução, o tempo de atendimento e a quantidade de sessões realizadas e de terapeutas que assistiram o paciente. Quanto à evolução do paciente, buscou-se também o motivo de desligamento ou abandono. Na terceira parte do protocolo, analisou-se os aspectos da avaliação de voz, por meio dos dados da avaliação perceptivo-auditiva. Os dados da caracterização vocal foram retirados dos registros da primeira avaliação de voz à qual o paciente foi submetido. Foram descritos os diferentes tipos de voz encontrados, o grau do desvio vocal, quando presente, e o tipo de disfonia.

Os dados dos prontuários foram analisados separadamente a fim de levantar as características da população com queixas e/ou alterações vocais atendida no ambulatório de fonoaudiologia. Além disso, algumas variáveis foram correlacionadas, buscando identificar os fatores interferentes na evolução do atendimento. A fim de avaliar a associação existente entre duas variáveis qualitativas, usou-se o teste de independência. A hipótese de independência entre variáveis foi escolhida quando o p-valor encontrado era inferior a $5 \%$. Ou seja, se $\mathrm{p}<0,05$, pôde-se dizer que as variáveis foram dependentes e que tiveram uma associação entre si. Análises de cruzamento verificaram relações entre as variáveis idade, profissão, escolaridade, procedência, tipo de voz, grau do desvio vocal e tipo de disfonia e a evolução do atendimento. Foram descritos também os valores mínimo e máximo da idade e os limites inferior e superior, com intervalo de $95 \%$ de confiança para média.

Em alguns protocolos havia variáveis sem registro, no entanto, esses protocolos não foram descartados a fim de se aproveitar os dados presentes, considerando-se os faltantes como não informados/não registrados. $\mathrm{Na}$ análise dos fa- 
tores que pudessem interferir na evolução do atendimento, foram considerados apenas os protocolos com dados completos de acordo com cada variável.

O presente estudo foi aprovado pelo Comitê de Ética e Pesquisa do Centro de Estudos da Voz (CEV) sob o número 25/2006 e da UFMG, sob o número 354/07.

\section{Resultados e discussão}

Num total de 963 prontuários presentes no arquivo do ambulatório de fonoaudiologia do Hospital das Clínicas da Universidade Federal de Minas Gerais, 336 representavam indivíduos com queixas e/ou alterações vocais, sendo enquadrados nos critérios de inclusão do estudo, e portanto, analisados. Este número representa 34,8\% do total de prontuários.

A população com queixas e/ou alterações vocais atendida no ambulatório de fonoaudiologia do Hospital das Clínicas da UFMG é composta principalmente por mulheres, com média de idade de 34,9 anos (Tabela 1). O predomínio de mulheres parece estar relacionado à característica feminina de buscar ajuda mais rapidamente e com maior frequência para os problemas de saúde do que os homens ${ }^{6}$. Acredita-se também que isso tenha acontecido pelo fato de a disfonia ser mais prevalente em mulheres ${ }^{7,8}$. A média de idade observada coincide com o período de maior demanda vocal das pessoas e, por isso, pode acarretar maior incidência de queixas e alterações relacionadas à voz ${ }^{6,8,9}$.

Em relação à profissão, escolaridade e procedência, houve predomínio de pessoas que não trabalham, com ensino fundamental incompleto e procedentes de Belo Horizonte, respectivamente (Tabela 1). O fato de haver maior quantidade de pessoas que não trabalham pode sugerir que as pessoas que trabalham tenham mais dificuldade em manter a assiduidade no tratamento. Um estudo, observou-se que algumas pessoas, embora necessitassem do tratamento, não tinham disponibilidade para continuá-lo com presença semanal ${ }^{10}$. Considerando a escolaridade, houve sujeitos em todas as categorias. Como os pacientes atendidos no ambulatório de fonoaudiologia do Hospital das Clínicas da Universidade Federal de Minas Gerais vêm obrigatoriamente de um encaminhamento do posto de saúde, supõe-se que esse fator não tenha tanta interferência no acesso aos serviços fonoaudiológicos. No entanto, alguns autores colocam que a falta de formação e informação pode ser o motivo da falta de procura pelos serviços de fonoaudiologia $^{11}$. Em relação à procedência dos pacientes, as pessoas que residem fora de Belo Horizonte só são encaminhadas para esta cidade se sua cidade de origem não dispuser do serviço público de fonoaudiologia. Assim, observa-se que a maioria das pessoas é de Belo Horizonte, ou seja, da mesma cidade onde se localiza o ambulatório. Atualmente, muitas cidades já dispõem de serviço público de fonoaudiologia, logo não é necessário que a população dessas cidades seja encaminhada para Belo Horizonte para realização de tratamento fonoaudiológico. Tal conduta vai ao encontro das diretrizes do SUS de proporcionar serviços de saúde próximos à população. Algumas pesquisas constataram também que a distância muito grande entre o local de atendimento e a residência dos pacientes, as dificuldades financeiras e a pouca disponibilidade de meios de transporte dificulta o acesso ${ }^{10,11}$.

Tabela 1. Distribuição de sexo, profissão, escolaridade, procedência e encaminhamento.

\begin{tabular}{|c|c|c|}
\hline Variáveis & $\mathbf{N}$ & $\%$ \\
\hline \multicolumn{3}{|l|}{ Sexo } \\
\hline Feminino & 231 & 68,8 \\
\hline Masculino & 105 & 31,2 \\
\hline \multicolumn{3}{|l|}{ Profissão } \\
\hline Profissional da voz & 68 & 20,2 \\
\hline Outra ocupação & 73 & 21,7 \\
\hline Não trabalha & 91 & 27,1 \\
\hline Não informada & 104 & 31,0 \\
\hline \multicolumn{3}{|l|}{ Escolaridade } \\
\hline Analfabeto & 1 & 0,3 \\
\hline Educação infantil & 2 & 0,6 \\
\hline Ensino fundamental incompleto & 41 & 12,2 \\
\hline Ensino fundamental completo & 5 & 1,5 \\
\hline Ensino médio incompleto & 13 & 3,9 \\
\hline Ensino médio completo & 24 & 7,1 \\
\hline Ensino superior incompleto & 10 & 3,0 \\
\hline Ensino superior completo & 9 & 2,7 \\
\hline Não informada & 231 & 68,8 \\
\hline \multicolumn{3}{|l|}{ Procedência } \\
\hline Belo Horizonte & 137 & 40,8 \\
\hline Grande Belo Horizonte & 31 & 9,2 \\
\hline Interior de Minas Gerais & 2 & 0,6 \\
\hline Outro estado & 0 & 0,0 \\
\hline Não informada & 166 & 49,4 \\
\hline \multicolumn{3}{|l|}{ Encaminhamento } \\
\hline Otorrinolaringologista & 181 & 53,9 \\
\hline Fonoaudiólogo & 22 & 6,5 \\
\hline Outra especialidade & 54 & 16,1 \\
\hline Não registrado & 79 & 23,5 \\
\hline
\end{tabular}


Quanto ao encaminhamento, a especialidade que mais encaminhou pacientes para atendimento em voz foi a otorrinolaringologia (Tabela 1). Acredita-se que este fato esteja relacionado à interface comum a estas áreas. Em estudos similares $^{6,8,10-12}$, observou-se que ambulatórios fonoaudiológicos infantis, que atendem alterações de fala e escrita, por exemplo, têm maior proximidade às escolas; as alterações de motricidade orofacial são predominantemente encaminhadas por ortodontistas; enquanto que as disfonias por otorrinolaringologistas.

Vários autores ${ }^{4,13,14}$ colocam que este tipo de caracterização da população de serviços públicos, ou seja, da realidade de cada unidade específica, é de extrema importância para o desenvolvimento de ações que levem em conta a dinamicidade das condições reais e de medidas de controles necessárias às instituições. $\mathrm{O}$ fato de trabalhar a partir de uma situação observada e de estabelecer a que ponto se deseja chegar com instrumentos e técnicas determinadas, possibilita a proposição de programas e de projetos melhor equacionados, permitindo uma avaliação quantitativa com base em comportamentos observáveis. Além disso, apesar dos programas de avaliação de dados não responderem a questões sobre a relação causal entre processo e resultado do tratamento, podem documentar tendências no tratamento de populações extensas ${ }^{9}$.

$\mathrm{O}$ atendimento foi realizado individualmente em $97,6 \%$ dos pacientes e não houve nenhuma ocorrência em grupo (Tabela 2). $\mathrm{O}$ atendimento individual é a prática mais utilizada para atendimento fonoaudiológico, sendo reproduzida no ambiente de clínica-escola e nos serviços públicos de saúde. Da amostra total, $47 \%$ receberam alta, $20,5 \%$ foram desligados e $32,4 \%$ abandonaram o tratamento (Tabela 1). A maior ocorrência de alta fonoaudiológica pode estar relacionada com o predomínio de disfonias funcionais e organofuncionais, situação nas quais o fonoaudiólogo tem um papel essencial na reabilitação ${ }^{8,15}$. Um estudo analisou os prontuários de 109 indivíduos com disfonia funcional submetidos a fonoterapia e constataram que ao final do tratamento, $82 \%$ da amostra apresentou resultados positivos ${ }^{7}$. Outros autores também estudaram os prontuários de 21 sujeitos com queixa de disfonia submetidos a fonoterapia e constataram que $50 \%$ dos pacientes abandonaram o tratamento ${ }^{8}$.

Em relação aos motivos de desligamento, o mais frequente foi a necessidade de encaminhamentos, e o maior motivo de abandono, em 62,4\% dos casos (Tabela 2), foi a falta sem justificativa.
O tratamento dos pacientes no ambulatório de voz é muito dinâmico, otimizando o processo terapêutico e, consequentemente, reduzindo a lista de espera. Em muitos casos, o paciente é convocado para o atendimento fonoaudiológico sem nunca ter se submetido a uma consulta por um otorrinolaringologista. A falta de um diagnóstico otorrinolaringológico limita o resultado da reabilitação vocal. Portanto, a conduta mais adequada nesses casos é o encaminhamento para a realização de um exame de laringe, o que aconteceu em $36,2 \%$ do total de pacientes desligados. No ambulatório de fonoaudiologia, o encaminhamento é feito para um profissional da rede pública de saúde, na qual, em geral, o paciente leva algum tempo para ser atendido. Neste caso, o paciente é desligado temporariamente até que seja visto por um otorrinolaringologista. As faltas não justificadas correspondem ao abandono, ou seja, os pacientes deixaram de frequentar os atendimentos sem notificar. O desconhecimento a respeito de como é feito o atendimento fonoaudiológico, a frequência necessária e a necessidade de treino em casa, pode ser levantado como hipótese para essa não aderência ao tratamento. A autopercepção de status de saúde pode afetar o comportamento dos indivíduos, fazendo com que eles acreditem ou não nas ações desenvolvidas. A valorização que o paciente dá à sua saúde, ao estresse, ao custo e à acessibilidade ao cuidado médico

Tabela 2. Características do atendimento.

\begin{tabular}{lrr}
\hline \multicolumn{1}{c}{ Variáveis } & N & \% \\
\hline Tipo de atendimento & & \\
Orientação & 8 & 2,4 \\
Individual & 328 & 97,6 \\
Grupo & 0 & 0,0 \\
Evolução do atendimento & & \\
Alta fonoaudiológica & 158 & 47,0 \\
Desligamento & 69 & 20,5 \\
Abandono & 109 & 32,4 \\
Motivo desligamento & & \\
Incompatibilidade de horário & 15 & 21,7 \\
Mudança de cidade & 4 & 5,8 \\
Encaminhamento & 25 & 36,2 \\
Não aderência & 12 & 17,4 \\
Outro & 8 & 11,6 \\
Não registrado & 5 & 7,2 \\
Motivo abandono & & \\
Falta sem justificativa & 68 & 62,4 \\
Dificuldade financeira & 4 & 3,7 \\
Outro & 9 & 8,3 \\
Não registrado & 28 & 25,7 \\
\hline
\end{tabular}


também influenciam na adesão a recomendações médicas, no caso, às orientações fonoaudiológi$\operatorname{cas}^{3}$. O processo de adoecer, receber e seguir conselhos e tratamentos médicos passa por uma série de estágios e são muitas as chances de nãoadesão, que se manifestam através de diferentes comportamentos: retardo em procurar ajuda de profissional especializado, recusa em participar de programas de promoção de saúde, comparecimento irregular às consultas marcadas, interrupção do seguimento médico com abandono às consultas e fracasso no seguimento das orientações prescritas ${ }^{16}$. Alguns autores colocam como causas de abandono desde razões particulares, financeiras ou greves da universidade a grandes distâncias entre o serviço e as residências, mudanças frequentes de endereço e de emprego, assim como a dificuldade em se ausentar do trabalho semanalmente ${ }^{8,10}$.

Pereira relata que vários pesquisadores descrevem suas experiências de sucesso com listas de espera e atendimentos em grupo. Os critérios utilizados para formação destes são: faixa etária, patologia e nível/grau de desenvolvimento. Os grupos são dinâmicos, podendo haver uma rotatividade de pacientes de acordo com a evolução terapêutica. $\mathrm{O}$ atendimento teve frequência semanal ${ }^{10}$.

Numa unidade básica de saúde (UBS), diante de uma lista de espera em crescimento contínuo, duas fonoaudiólogas criaram um grupo de atuação terapêutica, que procura oferecer um atendimento inicial à população que busca o serviço e que vem funcionando como porta de entrada para o serviço de fonoaudiologia 5 . Há autores que relataram a experiência de convocar os pacientes que estão na lista de espera e seus familiares a cada dois meses para participar de grupos de estimulação fonoaudiológica. Acreditam que este tipo de trabalho é fundamental para o controle da demanda ${ }^{17}$. Em outra situação, foi proposto atendimento em grupo, com duração de oito semanas e sessões semanais de 90 minutos ${ }^{4}$.

O tipo de voz predominante foi o combinado, seguido pelo rouca, sendo que, numa análise qualitativa, o tipo de voz combinado mais encontrado foi o rouco-soprosa. Nas vozes consideradas alteradas $(n=300)$, o grau de desvio vocal mais frequente foi o moderado (Tabela 3). Observa-se que na definição do tipo de voz houve uma quantidade significativamente menor de perda de dados em comparação ao tipo de disfonia. Isso sugere que o aluno-terapeuta prioriza mais a definição daquele atributo em detrimento deste. O grau de desvio vocal moderado pode estar relacionado à maior ocorrência de disfonias funcionais. Nessas disfonias, alguns autores colocam que as alterações vocais não são extremas a ponto de impedir a transmissão da mensagem ${ }^{15}$.

O tipo de disfonia não foi registrado em 281 prontuários, os quais correspondem a $83,6 \%$ da amostra (Tabela 3). Tal fato merece atenção, uma vez que o diagnóstico do paciente direciona a conduta terapêutica, podendo ser considerada a primeira etapa do próprio tratamento. Uma avaliação completa e precisa leva a um plano de reabilitação específico e individualizado para o paciente. Alguns autores afirmam que é indiscutível a superioridade de uma avaliação quando o médico e o fonoaudiólogo oferecem dados de suas áreas de conhecimento específico ${ }^{15,18,19}$. Este fato pode ser explicado, provavelmente, em função do aluno-terapeuta centrar mais sua atenção na manifestação vocal do paciente e, a partir desta, elaborar sua atuação terapêutica. Dentre os diagnósticos descritos $(n=55)$, predominou a disfonia funcional, distúrbio que tem o fonoaudiólogo como profissional de referência na, pois a disfonia decorre do próprio uso da voz e os re-

Tabela 3. Dados da avaliação vocal perceptivo-auditiva.

\begin{tabular}{lrr}
\hline \multicolumn{1}{c}{ Variáveis } & N & \multicolumn{1}{c}{$\%$} \\
\hline Tipo de voz & & \\
Neutra & 36 & 10,7 \\
Rouca & 96 & 28,6 \\
Soprosa & 11 & 3,3 \\
Tensa & 1 & 0,3 \\
Feminilizada & 2 & 0,6 \\
Tensa-estrangulada & 6 & 1,8 \\
Sussurrada & 4 & 1,4 \\
Trêmula & 1 & 0,3 \\
Infantilizada & 3 & 0,9 \\
Hipernasal & 1 & 0,3 \\
Virilizada & 1 & 0,3 \\
Combinado & 141 & 42,0 \\
Não registrado & 33 & 9,8 \\
Grau de desvio vocal & & \\
Leve & 93 & 31,0 \\
Moderado & 108 & 36,0 \\
Severo & 54 & 18,0 \\
Não se aplica & 36 & 10,7 \\
Não registrado & 45 & 15,0 \\
Tipo de disfonia & & \\
Disfonia funcional & 25 & 7,4 \\
Disfonia psicogênica & 7 & 2,1 \\
Disfonia organofuncional & 14 & 4,2 \\
Disfonia orgânica & 5 & 1,5 \\
Outro & 4 & 1,2 \\
Não registrado & 281 & 83,6 \\
\hline & & \\
& &
\end{tabular}


sultados terapêuticos dependem quase que exclusivamente de um trabalho vocal ${ }^{6,9,15}$.

Alguns autores, considerando as alterações vocais e laríngeas como distúrbio vocal, descrevem a ocorrência de disfonia funcional em 4,76\% da população ${ }^{8}$. Já outros, numa pesquisa em 5819 prontuários, encontraram uma incidência de $38 \%$ da amostra estudada ${ }^{20}$.

$\mathrm{O}$ atendimento em voz realizado com os pacientes teve duração média de seis meses, com 15 sessões . Cada um foi atendido por três terapeutas, em média, que atuaram juntos ou variaram a cada semestre curricular.

Segundo alguns autores, a terapia na presença de lesões benignas de prega vocal apresenta evolução evidente nos dois primeiros meses de tratamento, sendo que quando não há alterações estruturais mínimas associadas o resultado pode ser ainda mais rápido ${ }^{21}$. Um estudo descreve o tempo de terapia para disfonias que, no sexo masculino apresentou a média de atendimento de quatro meses e meio, enquanto que no sexo feminino, foi de 10 meses $^{8}$. No entanto, pela ocorrência de férias escolares e feriados e pelo fato de $o$ atendimento ser realizado por estudantes, ainda com pouca prática de atuação e aplicação dos conhecimentos teóricos, o tratamento pode ter sido prolongado.

Após esta análise inicial, determinando as características predominantes dos pacientes e do tratamento realizado, buscou-se identificar os fatores que podem interferir na evolução do atendimento fonoaudiológico. As análises feitas com variáveis em que houve grande perda de dados, como escolaridade, procedência e tipo de disfonia, têm menos fidedignidade que as demais. Não foram encontrados estudos que levem em consideração o efeito de diferentes variáveis na evolução do atendimento do paciente disfônico. Entretanto, considerou-se tal análise de grande importância, uma vez que, a discussão a respeito dos fatores que interferem no curso do tratamento traz informações relevantes sobre o servi- ço prestado e permitem propor eventuais modificações na atuação.

A evolução do atendimento de acordo com a idade foi apresentada na Tabela 4 . O valor de $\mathrm{p}$ $(0,027)$ indica que há uma diferença da distribuição da idade dos pacientes entre os tipos de evolução de atendimento. A faixa etária de pacientes que obtiveram alta fonoaudiológica é superior a de pacientes que foram desligados do tratamento ou abandonaram. Tal resultado pode apontar maior compromisso dos pacientes adultos com a terapia, pelo fato de necessitarem da voz para vida social e profissional e por perceberem com mais clareza a importância da fonoterapia como meio de melhora de sua alteração de voz. Além disso, estudos mostram que, nas crianças, em que a frequência e a evolução do tratamento dependem fundamentalmente da colaboração dos pais e que muitas vezes o encaminhamento é feito pela escola, os pais e os professores são pouco informados a respeito das disfonias infantis e tendem a valorizar mais alterações de fala, escrita e aprendizado ${ }^{10-12}$.

A evolução do atendimento de acordo com profissão, escolaridade, procedência, tipo de voz, grau de desvio vocal e tipo de disfonia está disposta na Tabela 5. Em relação à profissão, considerando a maneira como ela foi classificada, não há evidências estatísticas de que esta interfira na evolução do atendimento $(\mathrm{p}=0,141)$. A ausência de correlação entre essas variáveis pode estar relacionada à homogeneidade das três categorias propostas. Além disso, o ambulatório de fonoaudiologia atende pessoas independente de seu exercício profissional, não privilegiando profissionais da voz. Para a escolaridade, também não houve influência estatisticamente significante na evolução do atendimento $(\mathrm{p}=0,783)$. Vale notar também que as únicas categorias da escolaridade que apresentam mais de 20 observações são ensino fundamental incompleto e ensino médio completo, fato este que pode afetar o resultado do teste. Esses resultados confirmaram a hipótese anteri-

Tabela 4. Evolução do atendimento de acordo com a idade.

\begin{tabular}{lccccccc}
\hline \multicolumn{1}{c}{$\begin{array}{c}\text { Evolução do } \\
\text { atendimento }\end{array}$} & Média & $\begin{array}{c}\text { Limite } \\
\text { inferior }\end{array}$ & $\begin{array}{c}\text { Limite } \\
\text { superior }\end{array}$ & Mediana & $\begin{array}{c}\text { Desvio- } \\
\text { padrão }\end{array}$ & Mínimo & Máximo \\
\hline Alta fonoaudiológica & 36,88 & 33,74 & 40,03 & 34 & 19,66 & 5 & 82 \\
Desligamento & 29,58 & 25,09 & 34,07 & 26 & 18,54 & 4 & 76 \\
Abandono & 34,83 & 31,61 & 38,04 & 32 & 16,84 & 4 & 73 \\
\hline
\end{tabular}

Intervalo de 95\% de confiança para a média dos limites inferior e superior; $\mathrm{p}=0,027$. 
ormente levantada de que, como os pacientes atendidos no ambulatório de fonoaudiologia do Hospital das Clínicas da Universidade Federal de Minas Gerais vêm obrigatoriamente de um encaminhamento da UBS, a escolaridade parece ser um fator que não tenha tanta interferência no acesso aos serviços fonoaudiológicos da instituição e refutaram a afirmação de que a falta de formação e informação pode ser um motivo para a falta de procura pelos serviços de fonoaudiologia ${ }^{11}$.

$\mathrm{Na}$ análise da procedência (Tabela 5 ), novamente não houve associação estatisticamente significante $(\mathrm{p}=0,215)$. Observa-se que as porcentagens foram muito similares, exceto para os indivíduos do interior de Minas Gerais. Porém, esse grupo é composto por apenas dois indivíduos, o que inviabiliza qualquer comparação. Esperavase que os indivíduos procedentes da grande Belo Horizonte tivessem um índice de abandono maior, pela maior dificuldade de acesso ao serviço. No entanto, tal hipótese foi refutada pelos dados, uma vez que os pacientes de outras cidades são encaminhados para Belo Horizonte somente quando suas cidades de origem não têm serviço público de fonoaudiologia. A regionalização dos serviços favorece a adesão dos pacientes, pois estes não gastam tempo e dinheiro com transporte, conforme Pereira ${ }^{10}$.

Analisando-se a variável tipo de voz (Tabela 5), observa-se que há grande relação com a evo-

Tabela 5. Evolução do atendimento de acordo com profissão, escolaridade, procedência, tipo de voz, grau do desvio vocal e tipo de disfonia.

\begin{tabular}{|c|c|c|c|c|c|c|c|c|c|}
\hline \multirow[b]{2}{*}{ Variáveis } & \multicolumn{2}{|c|}{$\begin{array}{c}\text { Alta } \\
\text { fonoaudiológica }\end{array}$} & \multicolumn{2}{|c|}{ Desligamento } & \multicolumn{2}{|c|}{ Abandono } & \multicolumn{2}{|c|}{ Total } & \multirow[b]{2}{*}{$\mathrm{p}$} \\
\hline & $\mathbf{n}$ & $\%$ & $\mathbf{n}$ & $\%$ & $\mathbf{n}$ & $\%$ & $\mathbf{n}$ & $\%$ & \\
\hline Profissão & & & & & & & & & 0,141 \\
\hline Profissional da voz & 28 & 41,2 & 11 & 16,2 & 29 & 42,6 & 68 & 100 & \\
\hline Outra ocupação & 37 & 50,7 & 18 & 24,7 & 18 & 24,7 & 73 & 100 & \\
\hline Não trabalha & 42 & 46,2 & 24 & 26,4 & 25 & 27,5 & 91 & 100 & \\
\hline Escolaridade & & & & & & & & & 0,783 \\
\hline Analfabeto & 0 & 0,0 & 0 & 0,0 & 1 & 100 & 1 & 100 & \\
\hline Educação infantil & 1 & 50,0 & 0 & 0,0 & 1 & 50,0 & 2 & 100 & \\
\hline $\mathrm{EF}^{*}$ incompleto & 20 & 48,8 & 12 & 29,2 & 9 & 22,0 & 41 & 100 & \\
\hline EF completo & 4 & 80,0 & 0 & 0,0 & 1 & 20,0 & 5 & 100 & \\
\hline $\mathrm{EM}^{* *}$ incompleto & 4 & 30,8 & 4 & 30,8 & 5 & 38,5 & 13 & 100 & \\
\hline EM completo & 8 & 33,3 & 8 & 33,3 & 8 & 33,3 & 24 & 100 & \\
\hline $\mathrm{ES}^{* *}$ incompleto & 3 & 30 & 1 & 10,0 & 6 & 60,0 & 10 & 100 & \\
\hline ES completo & 6 & 66,7 & 2 & 22,2 & 1 & 11,1 & 9 & 100 & \\
\hline Procedência & & & & & & & & & 0,215 \\
\hline Belo Horizonte & 58 & 42,3 & 29 & 21,2 & 50 & 36,5 & 137 & 100 & \\
\hline Grande Belo Horizonte & 17 & 54,8 & 8 & 25,8 & 6 & 19,4 & 31 & 100 & \\
\hline Interior MG & 1 & 50,0 & 1 & 50,0 & 0 & 0,0 & 2 & 100 & \\
\hline Tipo de voz & & & & & & & & & 0,009 \\
\hline Neutra & 27 & 75,0 & 4 & 11,1 & 5 & 13,9 & 36 & 100 & \\
\hline Rouca & 39 & 40,6 & 23 & 24,0 & 34 & 35,4 & 96 & 100 & \\
\hline Soprosa & 6 & 54,5 & 2 & 18,2 & 3 & 27,3 & 11 & 100 & \\
\hline Combinado & 54 & 38,3 & 33 & 23,4 & 54 & 38,3 & 141 & 100 & \\
\hline Grau do desvio vocal & & & & & & & & & 0,564 \\
\hline Leve & 42 & 45,2 & 19 & 20,4 & 32 & 34,4 & 93 & 100 & \\
\hline Moderado & 47 & 43,5 & 26 & 24,1 & 35 & 32,4 & 108 & 100 & \\
\hline Severo & 19 & 35,2 & 13 & 24,1 & 22 & 40,7 & 54 & 100 & \\
\hline Tipo de disfonia & & & & & & & & & 0,007 \\
\hline Disfonia funcional & 20 & 80,0 & 2 & 8,0 & 3 & 12,0 & 25 & 100 & \\
\hline Disfonia psicogênica & 6 & 85,7 & 0 & 0,0 & 1 & 14,3 & 7 & 100 & \\
\hline Disfonia organo funcional & 15 & 35,7 & 8 & 57,1 & 1 & 7,1 & 14 & 100 & \\
\hline Disfonia orgânica & 2 & 40,0 & 1 & 20,0 & 2 & 40,0 & 5 & 100 & \\
\hline Outro & 3 & 75,0 & 0 & 0,0 & 1 & 25,0 & 4 & 100 & \\
\hline
\end{tabular}

*(EF) Educação Fundamental; ** (EM) Educação Média; ${ }^{* * *}$ (ES) Educação Superior. 
lução do atendimento $(\mathrm{p}=0,009)$. Para identificar onde estaria a associação entre as duas variáveis, a primeira foi dicotomizada e descobriu-se que a alta é mais frequente em pacientes que possuem tipo de voz neutra e soprosa $(\mathrm{p}=0,001)$. Já para o desligamento $(p=0,396)$ e para o abandono $(p=0,418)$, não foram encontradas associações significativas. É importante citar que a variável “tipo de voz" está dentre as que possuem amostra de tamanho maior, permitindo assim conclusões mais confiáveis. Os tipos de vozes neutra e soprosa, geralmente presentes em disfonias funcionais, têm boa evolução com o trabalho fonoaudiológico e garantem maior índice de alta terapêutica. O grau do desvio vocal, por sua vez, não teve associação com a evolução do atendimento $(\mathrm{p}=0,564)$. A relação talvez não tenha se evidenciado pelo fato de que as classes sejam semelhantes nos três tipos de evolução possíveis.

Em relação ao tipo de disfonia (Tabela 5), o pequeno valor de $\mathrm{p}(0,007)$ indica uma associação estatística com a evolução do atendimento. Novamente, a variável de evolução do atendimento foi dicotomizada. Os diagnósticos de disfonia funcional e disfonia psicogênica são as classes que mais estão associadas com a alta fonoaudiológica $(\mathrm{p}=0,026)$. Por outro lado, o diagnóstico de disfonia organofuncional é o que mais está ligado ao desligamento $(\mathrm{p}=0,004)$. Não há associação entre o diagnóstico fonoaudiológico e o abandono do atendimento $(p=0,351)$. Em geral, a maioria das pessoas não abandona o atendimento. É importante ressaltar que essa variável foi a que mostrou maior perda de dados, assim os resultados devem ser analisados com cautela. Pode-se considerar que esses achados se devem ao fato de que as disfonias funcionais e psicogênicas, quando não apresentam lesões laríngeas de base ou associadas, têm evolução terapêutica rápida e satisfatória, favorecendo a conclusão pelo paciente de todo o processo terapêutico até a alta fonoaudiológica.

Diante de todos os achados da pesquisa, alguns pontos podem ser refletidos frente às observações que foram descritas. É preciso modificar o pensamento de atuação tradicional e pensar em políticas mais eficientes para os serviços públicos de saúde, aplicando estudos que objetivem conhecer as pessoas que utilizam os serviços e que proponham formas de atendimento condizentes com as reais necessidades. Os serviços de saúde pública têm privilegiado as ações que visam resolver problemas de saúde da população, ao invés de investirem em programas que favoreçam a prevenção e a promoção da saúde.
Segundo Ramos ${ }^{22}$, tal situação é observada pela prática arraigada de atender apenas a demanda que se apresenta nos serviços de saúde.

Existem evidências científicas e clínicas de que indivíduos disfônicos se beneficiam dos serviços dos fonoaudiólogos. O investimento nas habilidades práticas dos alunos-terapeutas não será em vão no sentido de formar pessoas capazes de atender à demanda dos pacientes e da sociedade em relação às suas competências profissionais. Deve-se incentivar a elaboração e o preenchimento completo de um protocolo que constem todos os dados relevantes para identificação do paciente e do tratamento realizado. A preocupação com o atendimento da população impulsionou uma articulação mais estreita entre a formação acadêmica e a prestação de serviços, em uma via de mão dupla de trocas e apoios ${ }^{2}$. Isso fez com que o fonoaudiólogo assuma seu papel e lugar junto à promoção da saúde da população de maneira reflexiva, responsável e atuante ${ }^{23}$.

Apesar de a fonoaudiologia já ter ganho visibilidade em diversos segmentos sociais, ainda existem muitos caminhos a serem desbravados e reconhecidos. Dessa forma, vale a pena se pensar na orientação das pessoas que estão na lista de espera, não apenas acerca das patologias que apresentam, mas também sobre dados referentes a atuação fonoaudiológica, frequência desejada e tempo estimado, dentre outros aspectos. Ainda hoje, alguns pacientes por total desconhecimento do atendimento em fonoaudiologia, mostram-se surpresos quando informados sobre a frequência e duração média do tratamento ${ }^{10}$. Um dos grandes objetivos da promoção da saúde é influenciar mudanças nas questões da preservação da saúde e, para isso, é preciso contar com uma população que esteja informada e consciente do seu próprio processo saúde/doença. Neste contexto, a ação educativa torna-se fundamental para a estratégia de promoção da saúde ${ }^{24}$. Essa educação em saúde deve estar presente tanto nas salas de aula como na vivência do aluno como profissional.

\section{Conclusões}

Após a análise de 336 prontuários de indivíduos com queixas e/ou alterações vocais atendidos no ambulatório de fonoaudiologia do Hospital das Clínicas da Universidade Federal de Minas Gerais, no período de julho de 2003 a dezembro de 2006, pode-se observar que houve predominância de mulheres jovens, procedentes de Belo Horizonte, encaminhadas por um otorrinolaringo- 
logista, que não trabalham e têm formação até o ensino fundamental incompleto.

$\mathrm{O}$ atendimento realizado a esses pacientes aconteceu de forma individual, sendo que quase metade deles recebeu alta fonoaudiológica. O tipo de voz predominante foi o combinado, especialmente o rouco-soprosa, em grau moderado, e o tipo de disfonia mais comum foi a funcional.

Os indivíduos mais idosos, com disfonia funcional ou psicogênica e com voz do tipo neutra ou soprosa apresentaram a melhor correlação com a alta fonoaudiológica. Os indivíduos mais jovens e com disfonia organofuncional tiveram correlação estatística significativa com o desliga- mento. Já os indivíduos que evoluíram para abandono não apresentaram correlação com nenhuma das variáveis cruzadas.

A caracterização e avaliação de serviços públicos de saúde, como o ambulatório de fonoaudiologia do Hospital das Clínicas da Universidade Federal de Minas Gerais, mostrou que é possível estruturar melhor e assim buscar com mais eficácia formas de melhoria. Esse fato nos leva a refletir sobre as modificações que devemos promover para garantir um atendimento à população universal e igualitário, contribuindo assim para o aprimoramento direcionado a uma prática profissional de qualidade.

\section{Colaboradores}

LN Menezes participou da concepção, delineamento e redação do artigo, análise e interpretação de dados, e revisão do manuscrito. M Belhau e ACC Gama colaboraram na orientação geral e revisão do manuscrito. LC Teixeira trabalhou na revisão do manuscrito. 


\section{Referências}

1. Brasil. Constituição (1988). In: Paulo A, organizador. Constituição da República Federativa do Brasil. $18^{\text {a }}$ ed. Rio de Janeiro: DP\&A; 2005. p. 149-151.

2. Wechsler R. Universidade, serviços de saúde e a comunidade: o papel de programas de integração docente-assistenciais. In: Vieira RM, Vieira MM, Ávila CRB, Pereira LD. Fonoaudiologia e saúde pública. 2a ed. Carapicuíba: Pró-Fono; 2000. p. 45-54.

3. Acurcio FA, Guimarães MDC. Acesso a serviços e resultados na saúde de indivíduos infectados pelo HIV, em Belo Horizonte/MG, 1989-1994: uma abordagem quantitativa e qualitativa [tese]. Belo Horizonte: Universidade Federal de Minas Gerais; 1998.

4. Ortiz KZ, Bertachini L, Pereira LD. Atuação fonoaudiológica em unidades básicas de saúde. In: Vieira RM, Vieira MM, Ávila CRB, Pereira LD. Fonoaudiologia e saúde pública. $2^{\text {a }}$ ed. Carapicuíba: Pró-Fono; 2000. p. 121-136.

5. Gomes EMGP, Remencius NR. Fonoaudiologia na unidade básica de saúde. In: Lagrotta MM, César CPHAR. A Fonoaudiologia nas instituições. São Paulo: Lovise; 1997. p. 183-186.

6. Soar EGV. Caracterização de pacientes com disfonia atendidos em clínica fonoaudiológica particular [monografia]. São Paulo: Centro de Estudos da Voz; 2002.

7. Bridger MW, Epstein R. Functional voice disorders. A review of 109 patients. J Laryngol Otol 1983; 97(12):1145-1148.

8. Madazio GM, Cavicchia MI. Perfil do paciente disfônico da DERDIC [pesquisa de iniciação científica]. São Paulo: Pontifícia Universidade Católica de São Paulo; 1994.

9. Ramig LO, Verdolini K. Treatment efficacy: voice disorders. J Speech Lang Hear Res 1998; 41(1):S101S116.

10. Pereira MTJG. Fonoaudiologia: uma vivência em saúde pública [monografia]. São Paulo: Centro de Especialização em Fonoaudiologia Clínica/CEFAC; 1999.

11. Gonçalves CGO, Lacerda CBF, Perotino S, Mugnaine AMM. Demanda pelos serviços de fonoaudiologia no município de Piracicaba: estudo comparativo entre a clínica-escola e atendimento na prefeitura municipal. Pró-Fono 2000; 12(2):61-66.

12. César AM, Maksud SS. Caracterização da demanda de fonoaudiologia no serviço público municipal de Ribeirão das Neves - MG. Rev CEFAC 2007; 9(1):133-138.

13. Carvalho MS, Marzocchi KB. Avaliação da prática de vigilância epidemiológica nos serviços de saúde pública no Brasil. Rev Saude Publica 1992; 26(2):6674.
14. Mendes VLF. Fonoaudiologia e saúde coletiva: perspectivas de atuação nos serviços públicos de saúde. Dist Comun 1999; 10(2):213-224.

15. Behlau M, Madazio G, Feijó D, Pontes P. Avaliação de voz. In: Voz: o livro do especialista. v. 1. $2^{\text {a }}$ ed. Rio de Janeiro: Revinter; 2001. p. 85-247.

16. Rodrigues CS, Guimarães MDC, Acurcio FA. Fatores associados ao abandono do acompanhamento clínico por pacientes com infecção pelo HIV em um ambulatório público de referência, Belo Horizonte, 1993/ 1997 [dissertação]. Belo Horizonte: Universidade Federal de Minas Gerais; 2002.

17. Kerr FRM, Bertachini L, Ferrarini MAG, Reis DO. A sensibilização dos profissionais da saúde para com os distúrbios da comunicação humana. In: Lagrotta MM, César CPHAR. A fonoaudiologia nas instituições. São Paulo: Lovise; 1997. p. 187-192.

18. Bastian RW. Factors leading to successful evaluation and management of patients with voice disorders. Ear Nose Throat J 1988; 67(6):411-412.

19. Behrman A. Common practices of voice therapists in the evaluation of patients. J Voice 2005; 19(3):454469.

20. Perellò J, Miquel JA. Alteraciones de la voz. Barcelona: Científico-Médica; 1973. p. 116-117.

21. Behlau M, Madazio G, Feijó D, Azevedo R, Gielow I, Rehder MI. Aperfeiçoamento vocal e tratamento fonoaudiológico das disfonias. In: Belhau $\mathrm{M}$, organizador. Voz: o livro do especialista. v. 2. Rio de Janeiro: Revinter; 2005. p. 409-564.

22. Ramos L. Fonoaudiologia e saúde pública. Dist Comun 1991; 4(1):9-16.

23. Penteado RZ, Servilha EAM. Fonoaudiologia em saúde pública/coletiva: compreendendo prevenção e o paradigma da promoção da saúde. Dist Comun 2004; 16(1):107-116.

24. Marin CR, Chun RYS, Silva RC, Fedosse E, Leonelli BS. Promoção da saúde em fonoaudiologia: ações coletivas em equipamentos de saúde e de educação. Rev Soc Bras Fonoaudiol 2003; 8(1):35-41.

Artigo apresentado em 05/03/2009

Aprovado em 21/08/2009

Versão final apresentada em 01/09/2009 